\title{
Regulation of energy expenditure and substrate oxidation by short-chain fatty acids
}

\author{
Alia H Sukkar, Aaron M Lett, Gary Frost and Edward S Chambers \\ Section for Nutrition Research, Department of Medicine, Imperial College London, London, UK
}

Correspondence should be addressed to E S Chambers: e.chambers@imperial.ac.uk

\begin{abstract}
Short-chain fatty acids (SCFAs) are metabolites produced from the fermentation of dietary fibre by the gut microbiota. High-fibre diets have been associated with lower weight gain and a number of reports have therefore investigated if these positive effects of a dietary fibre on body weight can be replicated through the direct administration of SCFAs. Many of these studies have reported that SCFAs can prevent or attenuate long-term body weight gain by increasing energy expenditure through increased lipid oxidation. The aim of the present review is to therefore evaluate the current evidence for an effect of SCFAs on whole-body energy expenditure and to assess the potential underlying mechanisms. The available data highlights that SCFAs can exert multiple effects at various organ and tissue sites that would cumulatively raise energy expenditure via a promotion of lipid oxidation. In conclusion, the present review proposes that dietary interventions and other therapies that augment gut-derived SCFAs and systemic availability may present an effective strategy to improve long-term energy balance and body weight management.
\end{abstract}

\author{
Key Words \\ - short-chain fatty acids \\ - energy metabolism \\ - lipid oxidation \\ - obesity
}

\section{Introduction}

The prevalence of obesity has more than tripled since 1975 with $\sim 650$ million adults in the global population now classified as obese (a body mass index (BMI) $>30 \mathrm{~kg} / \mathrm{m}^{2}$ ). Many studies have identified that obesity is associated with a multitude of non-communicable diseases, such as cardiovascular disease, renal diseases, hypertension and type 2 diabetes (Whitlock et al. 2009). Although the causes of obesity are multifactorial, it is the result of a chronic positive energy balance where daily energy intake exceeds energy expenditure (Hill \& Peters 1998, Caballero 2007). Based on the average weight gain observed in early- and middle-aged adults ( $\sim 0.5 \mathrm{~kg} /$ year) (Williamson et al. 1990, Hebden et al. 2012), it is estimated that obesity can develop from a minor habitual positive energy balance ( $50 \mathrm{kcal} /$ day) (Hill et al. 2003). Theoretically, interventions to prevent this small positive energy balance, and thus incremental weight gain, need only promote a relatively small reduction in daily energy intake and/or enhancement in energy expenditure.

Long-term body weight management could be improved by an increased intake of dietary fibre, as prospective cohort studies have shown that high-fibre diets are associated with lower weight gain (Reynolds et al. 2019). For example, a study including 74,091 US females showed that during the 12-year follow-up period, a $12 \mathrm{~g} /$ day increase in dietary fibre intake per $1000 \mathrm{kcal} /$ day, was associated with $\sim 3.5 \mathrm{~kg}$ less weight gain (Liu et al. 2003). Similarly, intervention studies such as the Finish Diabetes Prevention study, which included 500 overweight middle-aged men and women with impaired glucose tolerance, showed that the treatment group who 
followed a high-fibre diet had sustained weight reduction $(>5 \%)$ after 3 years in comparison to the control group. Moreover, weight reduction was associated with fibre density in a dose-dependent manner where individuals who had ingested $<10 \mathrm{~g} / 1000 \mathrm{kcal}$ of fibre per day had a mean weight loss of $0.4 \mathrm{~kg}$ after 3 years in comparison to $3.0 \mathrm{~kg}$ in those who consumed $>15 \mathrm{~g} / 1000 \mathrm{kcal}$ of fibre per day (Lindström et al. 2006). Most countries recommend an average daily fibre intake of $25-35 \mathrm{~g} /$ day, however, the majority of individuals fail to reach this level. In the United Kingdom, for instance, average daily fibre intake is $13.6 \mathrm{~g} /$ day, which is almost half of the recommended $30 \mathrm{~g} /$ day for adults (Stephen et al. 2017).

A growing body of evidence suggests that the positive effects of dietary fibre on body weight gain may be due to the metabolites produced from microbial fermentation in the gut. The human gastrointestinal tract is host to around $10^{13}$ to $10^{14}$ bacteria with 2000 different species that contribute $>1 \mathrm{~kg}$ of our total body weight (Almeida et al. 2019). Short-chain fatty acids (SCFAs) are the major metabolic end products of gut microbial fermentation of dietary fibre with acetate, propionate and butyrate, generated in an approximate molar ratio of 60:20:20. Whilst acetate is synthesised in the liver following a prolonged fast or alcohol consumption, systemic SCFAs arise principally in mammals from the fermentation of dietary fibre in the gut (Scheppach et al. 1991). Acetate $\left(\mathrm{C}_{2} \mathrm{H}_{3} \mathrm{O}_{2}\right)$ is mainly generated via $\mathrm{CO}_{2}$ reductive methylation. Propionate $\left(\mathrm{C}_{3} \mathrm{H}_{5} \mathrm{O}_{2}\right)$ is produced through both acrylate and dicarboxylic pathways, depending on the type of propionic bacteria. Butyrate $\left(\mathrm{C}_{4} \mathrm{H}_{7} \mathrm{O}_{2}\right)$ is produced by the condensation and subsequent reduction of two molecules of acetyl-CoA molecules (Schönfeld \& Wojtczak 2016). Increasing dietary fibre intake has therefore been shown to promote production of SCFAs (Tilg et al. 2009, Byrne et al. 2015) and these gut-derived metabolites have been demonstrated to modulate metabolic pathways and receptor-mediated mechanisms at various tissue and organ sites (Gao et al. 2009, den Besten et al. 2015, Li et al. 2018). Specifically, SCFAs act as ligands for G-protein-coupled receptors (GPR) free fatty acid receptor 2 (FFAR2), FFAR3 and GPR109a which are expressed throughout the body and have been shown to regulate energy homeostasis (Kasubuchi et al. 2015). A number of research groups have therefore investigated the impact of administering SCFAs on weight gain to determine if the effects of a high-fibre diet can be replicated. Many of these studies have reported that administration of SCFAs promote improvements to long-term body weight by enhancing whole-body energy expenditure (Gao et al. 2009, Hattori et al. 2010, den Besten et al. 2015, Canfora et al. 2017, Chambers et al. 2018). For energy expenditure to increase these would need to be a corresponding elevation in substrate oxidation; principally an increased contribution of lipid and/or carbohydrate oxidation to 'fuel' the enhanced energy demands.

Thus, the aim of the present review is to (i) evaluate the impact of direct administration of SCFAs on energy expenditure and substrate oxidation in rodent and human studies and (ii) to assess the potential underlying mechanisms linking SCFAs with a regulatory role on energy expenditure and substrate oxidation.

\section{SCFAs administration increases whole-body energy expenditure and increases lipid oxidation in rodents}

Several studies have measured whole-body energy expenditure and substrate oxidation following acute and chronic oral supplementation of SCFAs in rodents. Hattori et al. showed that an acute oral administration of acetate acid $(1.5 \% 10 \mathrm{ml} / \mathrm{kg}$ of body weight (BW)) in mice increased energy expenditure and lipid oxidation in comparison to distilled water (Hattori et al. 2010). There were no differences in carbohydrate oxidation rates. Chronic daily oral injections of acetate are reported to have similar effects, as Yamashita et al. found that acetate treatment $(5.2 \mathrm{mg} / \mathrm{kg} \mathrm{BW})$ in comparison to distilled water reduced body weight at the end of 6 months and increased rates of whole-body oxygen consumption (Yamashita et al. 2009). Substrate oxidation rates were not described.

Other studies have observed that SCFAs promote similar chronic effects when incorporated into the diet. Gao et al. highlighted that supplementing butyrate $(5 \% \mathrm{w} / \mathrm{w})$ to a high-fat diet (HFD) for 16 weeks in mice prevented diet-induced obesity. The authors explained that this may be through an increase in energy expenditure via enhanced lipid oxidation, indicated by the lower respiratory exchange ratio (RER) (Gao et al. 2009). Similarly, den Besten et al. reported that a HFD supplemented with acetate, propionate or butyrate $(5 \%$ $\mathrm{w} / \mathrm{w})$ for 12 weeks, protected the mice from diet-induced obesity via increases in energy expenditure and a shift from carbohydrate oxidation to lipid oxidation, as seen by the lower RER (den Besten et al. 2015). Recently, Li et al. highlighted that after 1 week of butyrate supplementation $(5 \% \mathrm{w} / \mathrm{w})$ energy expenditure was unaffected; yet, rates of 
lipid oxidation were elevated and carbohydrate oxidation lowered (Li et al. 2018).

In addition, other rodent studies have examined the impact of SCFAs administration on energy metabolism following intraperitoneal infusion. In the acute term, Kimura et al. has shown that propionate can increase oxygen consumption in comparison to control when given intraperitoneally at a rate of $1 \mathrm{~g} / \mathrm{kg}$ BW (Kimura et al. 2011). Substrate oxidation rates were not reported. Sahuri-Arisolyu et al. observed that when acetate was administered intraperitoneally to mice fed a HFD for a period of 6 weeks, there was a significant decrease in whole-body adiposity. The acetate administered mice showed no differences in food intake, but had an elevated heat production, which would indicate increased energy expenditure (Sahuri-Arisoylu et al. 2016).

In summary, the majority of evidence highlights that acute and chronic provision of SCFAs in rodents promotes energy expenditure whether administered orally or via intraperitoneal infusion. Where measured, the increase in energy expenditure is accompanied by an elevation in the rates of lipid oxidation.

\section{SCFAs administration increases whole-body energy expenditure and increases lipid oxidation in humans}

Only one human study has investigated the acute effect of oral administration of SCFAs on whole-body resting energy expenditure and substrate oxidation. Recently, Chambers et al. showed that acute oral sodium propionate ingestion $(6.8 \mathrm{~g})$ increased both resting energy expenditure and lipid oxidation in 18 healthy male $(n=9)$ and female $(n=9)$ volunteers compared to a sodium chloride control (Chambers et al. 2018). A single study has also examined the chronic effect of oral SCFAs supplementation in humans on energy expenditure. Bouter et al. report in a pilot study of nine healthy lean males and ten obese males with metabolic syndrome that oral sodium butyrate supplementation ( $4 \mathrm{~g} /$ day) for 4 weeks had no significant effect on resting energy expenditure (Bouter et al. 2018). The potential impact on substrate oxidation was not reported.

Human trials have shown comparable results with rodent studies when SCFAs are administered via direct infusion to the gut. In a randomised double-blind crossover trial, Van der Beek et al. demonstrated that an infusion of sodium acetate to the distal colon $(180 \mathrm{mM})$ in overnight fasted overweight and obese men increased lipid oxidation in comparison to a sodium chloride placebo. There were no observed changes in rates of resting energy expenditure (van der Beek et al. 2016). Also, Canfora et al. demonstrated in 12 overnight fasted normoglycemic men that rectally infused mixtures of physiological quantities of SCFAs $(200 \mathrm{mM})$ stimulate lipid oxidation and decreased carbohydrate oxidation, while highacetate (sodium acetate (60\%), sodium propionate $(20 \%)$, sodium butyrate (20\%)) and high-propionate infusions (sodium acetate (45\%), sodium propionate $(35 \%)$, sodium butyrate (20\%)) enhanced resting energy expenditure in comparison to the sodium chloride placebo. However, no significant differences between interventions were found in energy expenditure or substrate oxidation in the postprandial state following ingestion of standard oral glucose tolerance test (Canfora et al. 2017).

In summary, although most human studies show similarities with rodent investigations that SCFAs can increase energy expenditure via increases in lipid oxidation, it is noteworthy that human trials are presently scarce, and more interventional studies are needed to confirm the impact of acute and chronic SCFAs administration on whole-body energy expenditure and substrate oxidation.

\section{SCFAs bioavailability and contribution to energy requirements}

When assessing potential mechanisms linking SCFAs with a regulatory role on energy expenditure and substrate oxidation it is important to consider the bioavailabilities of gut-derived SCFAs at different organ sites and their contribution to energy metabolism.

Total concentration of SCFAs in the human colon is $\sim 200 \mathrm{mM}$ with the greatest amount present in the cecum (Cummings et al. 1987). Butyrate is preferentially oxidised by colonocytes as an energy substrate (Clausen $\&$ Mortensen 1995). Acetate and propionate may also be metabolised by colonocytes, but to a much smaller extent. The SCFAs not metabolised by the gut are absorbed into the portal vein with those SCFAs not extracted by the liver entering the hepatic vein and peripheral circulation. Marked differences in SCFAs concentrations are seen between the portal and hepatic vein, with $\sim 80 \%$ of propionate and butyrate in the portal vein taken-up by the liver compared with $\sim 40 \%$ acetate (Cummings et al. 1987, Bloemen et al. 2009, Neis et al. 2019). Consequently, acetate is the only gut-derived SCFAs present in appreciable https://joe.bioscientifica.com

https://doi.org/10.1530/JOE-19-0098
(C) 2019 Society for Endocrinology Published by Bioscientifica Ltd.
Printed in Great Britain 
amounts ( $>50 \mu \mathrm{mol}$ ) in peripheral blood (Cummings et al. 1987, Bloemen et al. 2009, Neis et al. 2019), which is imperative when assessing the potential effects of SCFAs on metabolic regulation at different organ sites.

In mammalian species, all three SCFAs are available as precursors for lipid or carbohydrate synthesis or can be oxidised in the TCA (tricarboxylic acid) cycle. Acetate and butyrate enter the TCA cycle as acetylCoA (acetyl coenzyme A), which may also be used as a substrate for de novo lipogenesis. Propionate enters the TCA cycle as succinyl-CoA, which can be used as a precursor for hepatic gluconeogenesis. The maximum ATP (adenosine triphosphate) yield from complete oxidation of acetate, propionate and butyrate is 10,18 and $27 \mathrm{ATP} / \mathrm{M}$, equivalent to $73,131.4$ and $197.1 \mathrm{kcal} / \mathrm{M}$, respectively (Baldwin 1995). Gut-derived SCFAs are therefore considered important substrates for energy metabolism and are estimated to contribute $\sim 5-10 \%$ of human daily energy requirements (Bergman 1990). In the rodent and human studies reporting an increase in energy expenditure and lipid oxidation rates following SCFAs administration, the SCFAs intervention has not been compared against an energy-matched control. It has been proposed in these studies that the increase in energy expenditure and lipid oxidation is due to SCFAs stimulating the utilisation of endogenous energy sources (Gao et al. 2009, Hattori et al. 2010, den Besten et al. 2015, Canfora et al. 2017, Chambers et al. 2018). However, it is plausible that a proportion of the recorded increase in whole-body energy expenditure could be attributed to the oxidation of the exogenous SCFAs and/or the energy cost of utilising the SCFAs for lipid or carbohydrate synthesis. Future work is therefore needed to differentiate the contribution of the administered SCFAs and endogenous energy sources to the increase in energy expenditure and lipid oxidation rates observed following SCFAs supplementation.

\section{The impact of SCFAs on hepatic energy metabolism}

As previously stated, a considerable proportion of the three principal SCFAs absorbed from the gut are extracted and metabolised by the liver (Cummings et al. 1987, Bloemen et al. 2009, Neis et al. 2019). It would therefore be logical that the increase in wholebody energy expenditure recorded following SCFAs administration would be due to alterations in hepatic metabolic processes.

(C) 2019 Society for Endocrinology Published by Bioscientifica Ltd. Printed in Great Britain
Den Besten et al. showed that mice were protected from hepatic steatosis by dietary supplementation of SCFAs over a period of 12 weeks. One of the possible underlying mechanisms was the decrease in peroxisome proliferator-activated receptor gamma (Pparg) activity in the liver. Pparg increases energy expenditure and serves as a metabolic switch from lipid synthesis to oxidation by increasing the AMP (adenosine monophosphate):ATP ratio and mitochondrial uncoupling protein 2 (UCP2) expression via AMPK (5' adenosine monophosphateactivated protein kinase). Indeed, by using liver-specific Pparg-knockout (KO) mice, the SCFAs mediated increase in hepatic lipid oxidation and decrease in hepatic steatosis were abolished, thus confirming the proposed mechanism (den Besten et al. 2015).

Gao et al. also showed that butyrate can stimulate energy expenditure in mice fed a HFD by modulating liver metabolism. Liver culture cells of butyrate-treated mice had increased phosphorylation of AMPK and p38 and expression of Ppargc1a (peroxisome proliferatoractivated receptor gamma, coactivator 1 alpha), which would supress hepatic lipid storage in favour of oxidation (Gao et al. 2009).

In addition, Sahuri-Arisoylu showed that acetate administration can decrease intrahepatocellular lipid accumulation and improve liver function in rodents via different mechanisms. Acetate supressed the expression of several genes in the liver involved with de novo lipogenesis such as sterol regulatory elementbinding protein 1 (Srebf1), fatty acid synthase (Fasn) and acetyl-CoA carboxylase (Acaca), thereby decreasing lipid accumulation. Furthermore, acetate increased the expression of complexes III, IV and V in the electron transport chain of hepatic mitochondria, which would improve mitochondrial efficiency and elevate ATPgenerating capacity (Sahuri-Arisoylu et al. 2016).

Finally, Kondo et al. also showed that acetate can protect against diet-induced obesity in mice and promote lipid oxidation via changes in liver metabolism. The acetate-treated rodents had higher mRNA expression of peroxisome-proliferator-activated receptor alpha (Ppara) via activation of AMP-activated, alpha 2 catalytic subunit (Prkaa2) in hepatocytes. This metabolic response increased fatty acid oxidation enzymes such as acyl-CoA oxidase (ACO) and carnitine palmitoyl transferase-1 (CPT1), as well as the thermogenic UCP2 (Kondo et al. 2009).

In summary, studies have reported that SCFAs promote metabolic changes in the liver that would upregulate lipid oxidation and energy expenditure. 


\section{The impact of SCFAs on adipose tissue energy metabolism}

SCFAs have also been shown to induce profound changes in adipose tissue metabolism that would explain the changes in energy expenditure and lipid oxidation observed following SCFAs supplementation.

In white adipose tissue (WAT), den Besten et al. showed that mice were protected from HFD-induced obesity and its associated metabolic abnormalities via downregulation of Pparg and the subsequent increase in UCP2 expression and fatty acid oxidation, similar to that found in hepatic tissue. To confirm this mechanism, adipose tissue-specific Pparg-KO mice and wild-type mice were compared, and indeed, the effects of SCFAs on BW gain, WAT mass and adipose-specific lipid oxidation were eliminated in the $\mathrm{KO}$ mice, thus confirming the role of Pparg in mediating these effects (den Besten et al. 2015).

SCFAs have also been shown to increase the lipid-buffering capacity of WAT. In vitro, Jocken et al. showed a decrease in intracellular lipolysis in human adipocytes using different physiological $(1 \mu \mathrm{mol} / \mathrm{L})$ and supraphysiological $(1 \mathrm{mmol} / \mathrm{L})$ concentrations of SCFAs mixtures. Moreover, acetate, which is the most abundant SCFAs in systemic circulation, appears to have the most profound effect via decreasing the phosphorylation of hormone-sensitive lipase. Further investigation revealed that this antilipolytic effect is mainly mediated by FFAR2 and/or FFAR3 expressed on WAT (Jocken et al. 2017).

Although SCFAs have been shown to reduce lipolysis and promote lipid oxidation in WAT, WAT in itself is not classified as a highly metabolic active tissue. Thus, it is questionable that the effects of SCFAs on energy expenditure or lipid metabolism are driven by metabolic changes in WAT. A more likely mechanism is the potential effects of SCFAs on 'metabolically active' brown adipose tissue (BAT) or the beiging of WAT (Carpentier et al. 2018).

Indeed, Lu et al. demonstrated that via regulation of FFAR 2/3, SCFAs can protect against HFD induced obesity by promoting beiging of WAT, which would increase energy expenditure and fatty acid oxidation. Mice fed an HFD supplemented with SCFAs had higher expression of genes related to mitochondrial biogenesis, including Ppargc1a, as well as specific gene markers for beige adipocytes involved in fat oxidation. Also, the mRNA levels of rate-limiting enzymes involved in fatty acid oxidation (CPT1A, CPT1C and CPT2) in WAT were increased in comparison to the HFD control mice (Lu et al. 2016).

Sahuri-Arisoylu et al. also showed that mice fed a HFD treated with acetate had increased heat production.
This coincided with increased expression of UCP1 in subcutaneous WAT together with increases in PR domain containing 16 (Prdm16), which is involved in the differentiation of brown-like adipocytes and thus increases the thermogenic potential via beiging of WAT (Sahuri-Arisoylu et al. 2016).

The effect of acetate on BAT activity and WAT beiging is supported by Yamashita et al. (2009) who demonstrated that acetate administered mice had $\sim 1.5$ times higher transcript levels of lipolytic genes, such as LCACD (longchain acyl-CoA dehydrogenase), 3KACT (3-ketoacylCoA thiolase) and peroxisome proliferator activator receptor delta (Ppard) in BAT and WAT in comparison to water-administered mice. Moreover, histological analysis revealed smaller lipid droplets in BAT and WAT after acetate administration, which reflects a higher thermogenic capacity (Yamashita et al. 2009).

Butyrate has been reported to have similar effects, as Gao et al. also showed that butyrate-treated mice having greater adaptation to cold exposure in comparison to control mice. Indeed, this was related to enhanced thermogenesis by BAT, where the mRNA of two prominent thermogenesis-related genes Ppargc1a and Ucp1 were increased (Gao et al. 2009). Also, Li et al. recently showed that butyrate can have an effect on BAT thermogenic activity via an increase in sympathetic outflow to BAT, as mice fed an HFD supplemented with butyrate had increased levels of UCP1 within BAT, which significantly enhanced thermogenic capacity. However, butyrate administration did not induce beiging of WAT (Li et al. 2018).

The impact of SCFAs on BAT activity and WAT beiging is an attractive mechanism to explain the effects of SCFAs on energy metabolism; however, these outcomes are yet to be demonstrated in humans. Indeed, oral butyrate administration was found to have no effects on BAT activity in human subjects (Bouter et al. 2018). Whilst the presence of BAT has been established in adult humans, the direct translation of findings from rodents to humans is a topic of debate, primarily due to species differences in the anatomical location of BAT, the relative amounts of BAT and the existence of WAT beiging in humans (Nedergaard et al. 2007, Carpentier et al. 2018).

\section{The impact of SCFAs on skeletal muscle energy metabolism}

Studies have also shown that SCFAs can modulate metabolic responses in skeletal muscle that would promote changes in energy expenditure and substrate oxidation. 
Yamashita et al. reported that acetate-treated mice had significantly higher transcription levels of myoglobin, GLUT4 (glucose transporter type 4) and KLF15 (Kruppel like factor 15) and genes in skeletal muscle, which are all involved in regulating energy metabolism. Moreover, the ingested acetate increased the AMP:ATP ratio thereby promoting the phosphorylation of AMPK which regulates numerous enzymes involved with lipid metabolism (Yamashita et al. 2009). A recent in vitro study supports the finding that acetate promotes the phosphorylation of AMPK and its downstream metabolic pathways in skeletal muscle (Maruta et al. 2016).

Gao et al. showed several changes in skeletal muscle tissue following butyrate supplementation. It was found that butyrate promoted the development of type 1 slowtwitch skeletal muscle fibres, which are characterised by enhanced mitochondrial density and capacity for lipid oxidation. This metabolic adaptation was associated with increased expression of Ppargc1a target genes, such as Cpt1b and $m t$-Co1 (cytochrome c oxidase I) and Ppard. Further analysis showed that these changes may be due to decreases in histone deacetylase which regulate gene transcription found in the butyrate-treated group (Gao et al. 2009).

An investigation using $\mathrm{C} 2 \mathrm{C} 12$ muscle cells has also highlighted that propionate was effective at increasing the gene and protein expression of UCP1, which would increase the thermogenic capacity and energy expenditure in skeletal muscle cells (Murakami et al. 2015).

\section{Other potential mechanisms}

Studies have reported an important role of the vagal and sympathetic nervous system mediating the positive effects of SCFAs on energy expenditure. For instance, Li et al. showed that the butyrate-mediated effect on BAT was through a 'gut-brain' neural circuit since mice receiving subdiaphragmatic vagotomy no longer exhibited an increase in BAT thermogenic capacity following butyrate administration (Li et al. 2018). Furthermore, Kimura et al . showed that propionate can directly induce sympathetic outflow via FFAR3 expressed at the level of the sympathetic ganglion. Moreover, they were able to demonstrate that

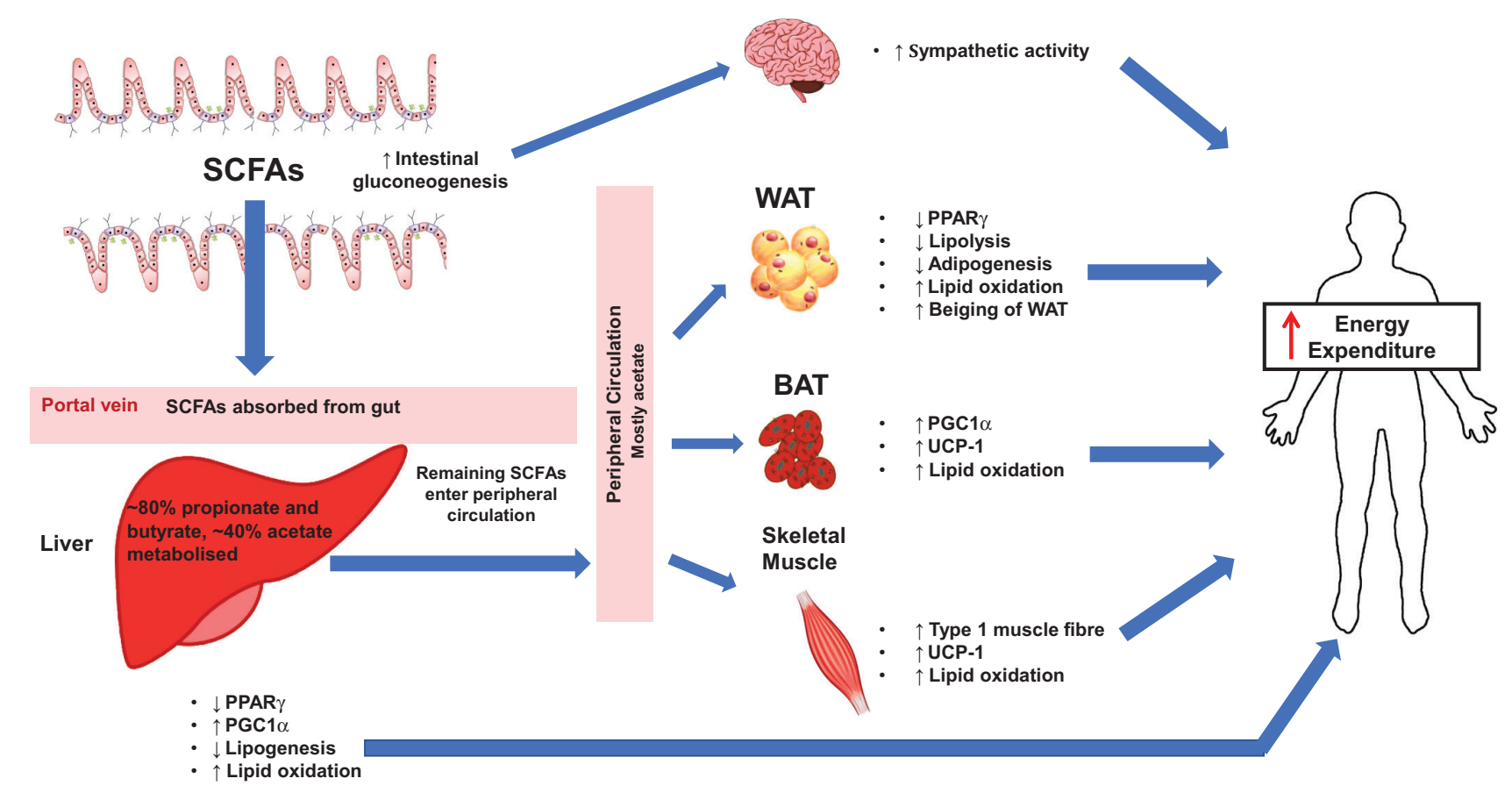

Figure 1

An overview of the effect of SCFAs on energy metabolism and lipid oxidation at different organ sites. SCFAs are produced in the gut from the fermentation of dietary fibre by the gut microbiota. Propionate and butyrate can induce intestinal gluconeogenesis, which enhances energy expenditure via gut-neural signalling. SCFAs are absorbed from the gut lumen and enter the portal vein where $\sim 80 \%$ propionate and butyrate and $\sim 40 \%$ of acetate are metabolised in the liver. In the liver, SCFAs promote lipid oxidation and reduce lipogenesis. The remaining SCFAs (predominantly acetate) enter the peripheral circulation. In WAT, SCFAs increase adipogenesis and fatty acid oxidation and reduce lipolysis. Beige adipogenesis of WAT is also upregulated. In BAT, SCFAs increase thermogenic activity. In skeletal muscle, SCFAs promote the development of type-1 muscle fibres and enhance capacity for lipid oxidation. 
propionate can increase oxygen consumption and thus energy expenditure via FFAR3, as this effect was abolished in FFAR3-KO mice (Kimura et al. 2011). This mechanism may partly explain the findings of Bellahcene et al. who showed that male FFAR3-KO mice had a higher body fat content and decreased energy expenditure (Bellahcene et al. 2013).

Finally, De Vadder et al. proposed that the beneficial effects of SCFAs on energy homeostasis are mediated through intestinal gluconeogenesis (IGN). In this investigation a propionate or butyrate-enriched diet $(5 \% \mathrm{wt} / \mathrm{wt})$ reduced $\mathrm{BW}$ gain in rats after 10 days. This improvement in BW was not associated with changes in energy intake, indicating that SCFAs treatment would have chronically enhanced energy expenditure. It was found that propionate can induce IGN via 'gut-brain' neural communication involving FFAR3 expressed in the portal vein. Butyrate was shown to enhance IGN by increasing cAMP, a potent activator of gluconeogenic genes, namely G6pc (glucose-6-phosphatase) and Pck1. Indeed, KO mice for these intestinal gluconeogenic genes were no longer protected from diet induced obesity with SCFAs treatment (De Vadder et al. 2014).

\section{Conclusions}

The present review proposes that SCFAs exert multiple effects on various organ and tissue sites that would cumulatively promote lipid oxidation and energy expenditure. These proposed mechanisms are summarised in Fig. 1. The majority of the available mechanistic evidence to support a role of SCFAs in energy metabolism has been obtained from in vitro experiments and rodent models. Furthermore, rodent models have commonly relied on RER to infer changes in substrate oxidation, rather than quantified rates of lipid and carbohydrate oxidation. Additional data from human studies to strengthen the proposed mechanisms are also warranted, as a direct translation of experimental outcomes from rodents may be limited. Should these metabolic effects be demonstrated in humans, it would support the design of dietary interventions and other therapies to augment SCFAs production and systemic availability as a strategy to improve long-term energy balance and weight management.

\section{Declaration of interest}

The authors declare that there is no conflict of interest that could be perceived as prejudicing the impartiality of this review.
(C) 2019 Society for Endocrinology Published by Bioscientifica Ltd. Printed in Great Britain

\section{Funding}

The Section of Endocrinology and Investigative Medicine is funded by grants from the MRC, BBSRC, NIHR, an Integrative Mammalian Biology (IMB) Capacity Building Award, an FP7-HEALTH-2009-241592 EuroCHIP grant and is supported by the NIHR Biomedical Research Centre Funding Scheme. The views expressed are those of the author(s) and not necessarily those of the NHS, the NIHR or the Department of Health.

\section{Author contribution statement}

A H S, A M L and E S C searched and reviewed the literature. All authors contributed to the final content of the paper.

\section{References}

Almeida A, Mitchell AL, Boland M, Forster SC, Gloor GB, Tarkowska A, Lawley TD \& Finn RD 2019 A new genomic blueprint of the human gut microbiota. Nature 568 499-504. (https://doi.org/10.1038/s41586019-0965-1)

Baldwin RL 1995 Modeling Ruminant Digestion and Metabolism. Berlin, Germany: Springer Science \& Business Media.

Bellahcene M, O'Dowd JF, Wargent ET, Zaibi MS, Hislop DC, Ngala RA, Smith DM, Cawthorne MA, Stocker CJ \& Arch JR 2013 Male mice that lack the G-protein-coupled receptor GPR41 have low energy expenditure and increased body fat content. British Journal of Nutrition 109 1755-1764. (https://doi.org/10.1017/S0007114512003923)

Bergman EN 1990 Energy contributions of volatile fatty acids from the gastrointestinal tract in various species. Physiological Reviews 70 567-590. (https://doi.org/10.1152/physrev.1990.70.2.567)

Bloemen JG, Venema K, van de Poll MC, Damink SWO, Buurman WA \& Dejong CH 2009 Short chain fatty acids exchange across the gut and liver in humans measured at surgery. Clinical Nutrition 28 657-661. (https://doi.org/10.1016/j.clnu.2009.05.011)

Bouter K, Bakker GJ, Levin E, Hartstra AV, Kootte RS, Udayappan SD, Katiraei S, Bahler L, Gilijamse PW, Tremaroli V, et al. 2018 Differential metabolic effects of oral butyrate treatment in lean versus metabolic syndrome subjects. Clinical and Translational Gastroenterology 9155. (https://doi.org/10.1038/s41424-018-0025-4)

Byrne CS, Chambers ES, Morrison DJ \& Frost G 2015 The role of short chain fatty acids in appetite regulation and energy homeostasis. International Journal of Obesity 39 1331-1338. (https://doi.org/10.1038/ijo.2015.84)

Caballero B 2007 The global epidemic of obesity: an overview. Epidemiologic Reviews 29 1-5. (https://doi.org/10.1093/epirev/mxm012)

Canfora EE, van der Beek CM, Jocken JWE, Goossens GH, Holst JJ, Damink SWM, Lenaerts K, Dejong CHC \& Blaak EE 2017 Colonic infusions of short-chain fatty acid mixtures promote energy metabolism in overweight/obese men: a randomized crossover trial. Scientific Reports 7 2360. (https://doi.org/10.1038/s41598-01702546-x)

Carpentier AC, Blondin DP, Virtanen KA, Richard D, Haman F \& Turcotte ÉE 2018 Brown adipose tissue energy metabolism in humans. Frontiers in Endocrinology 9 447. (https://doi.org/10.3389/ fendo.2018.00447)

Chambers ES, Byrne CS, Aspey K, Chen Y, Khan S, Morrison DJ \& Frost G 2018 Acute oral sodium propionate supplementation raises resting energy expenditure and lipid oxidation in fasted humans. Diabetes, Obesity and Metabolism 20 1034-1039. (https://doi.org/10.1111/ dom.13159)

Clausen MR \& Mortensen PB 1995 Kinetic studies on colonocyte metabolism of short chain fatty acids and glucose in ulcerative colitis. Gut 37 684-689. (https://doi.org/10.1136/gut.37.5.684)

Cummings JH, Pomare EW, Branch WJ, Naylor CP \& Macfarlane GT 1987 Short chain fatty acids in human large intestine, portal, hepatic and venous blood. Gut 28 1221-1227. (https://doi.org/10.1136/ gut.28.10.1221) 
De Vadder F, Kovatcheva-Datchary P, Goncalves D, Vinera J, Zitoun C, Duchampt A, Bäckhed F \& Mithieux G 2014 Microbiota-generated metabolites promote metabolic benefits via gut-brain neural circuits. Cell 156 84-96. (https://doi.org/10.1016/j.cell.2013.12.016)

den Besten G, Bleeker A, Gerding A, van Eunen K, Havinga R, van Dijk TH, Oosterveer MH, Jonker JW, Groen AK, Reijngoud DJ, et al. 2015 Shortchain fatty acids protect against high-fat diet-induced obesity via a PPAR $\gamma$-dependent switch from lipogenesis to fat oxidation. Diabetes 64 2398-2408. (https://doi.org/10.2337/db14-1213)

Gao Z, Yin J, Zhang J, Ward RE, Martin RJ, Lefevre M, Cefalu WT \& Ye J 2009 Butyrate improves insulin sensitivity and increases energy expenditure in mice. Diabetes 58 1509-1517. (https://doi. org/10.2337/db08-1637)

Hattori M, Kondo T, Kishi M \& Yamagami K 2010 A single oral administration of acetic acid increased energy expenditure in C57BL/6J mice. Bioscience, Biotechnology, and Biochemistry 74 2158-2159. (https://doi.org/10.1271/bbb.100486)

Hebden L, Chey T \& Allman-Farinelli M 2012 Lifestyle intervention for preventing weight gain in young adults: a systematic review and meta-analysis of RCTs. Obesity Reviews 13 692-710. (https://doi. $\operatorname{org} / 10.1111 / j .1467-789 X .2012 .00990 . x)$

Hill JO \& Peters JC 1998 Environmental contributions to the obesity epidemic. Science 280 1371-1374. (https://doi.org/10.1126/ science.280.5368.1371)

Hill JO, Wyatt HR, Reed GW \& Peters JC 2003 Obesity and the environment: where do we go from here? Science 299 853-855. (https://doi.org/10.1126/science.1079857)

Jocken JWE, González Hernández MA, Hoebers NTH, van der Beek CM, Essers YPG, Blaak EE \& Canfora EE 2017 Short-chain fatty acids differentially affect intracellular lipolysis in a human white adipocyte model. Frontiers in Endocrinology 8 372. (https://doi.org/10.3389/ fendo.2017.00372)

Kasubuchi M, Hasegawa S, Hiramatsu T, Ichimura A \& Kimura I 2015 Dietary gut microbial metabolites, short-chain fatty acids, and host metabolic regulation. Nutrients 7 2839-2849. (https://doi. org/10.3390/nu7042839)

Kimura I, Inoue D, Maeda T, Hara T, Ichimura A, Miyauchi S, Kobayashi M, Hirasawa A \& Tsujimoto G 2011 Short-chain fatty acids and ketones directly regulate sympathetic nervous system via $\mathrm{G}$ protein-coupled receptor 41 (GPR41). PNAS 108 8030-8035. (https:// doi.org/10.1073/pnas.1016088108)

Kondo T, Kishi M, Fushimi T \& Kaga T 2009 Acetic acid upregulates the expression of genes for fatty acid oxidation enzymes in liver to suppress body fat accumulation. Journal of Agricultural and Food Chemistry 57 5982-5986. (https://doi.org/10.1021/jf900470c)

Li Z, Yi CX, Katiraei S, Kooijman S, Zhou E, Chung CK, Gao Y, van den Heuvel JK, Meijer OC, Berbée JFP, et al. 2018 Butyrate reduces appetite and activates brown adipose tissue via the gut-brain neural circuit. Gut 67 1269-1279. (https://doi.org/10.1136/gutjnl-2017-314050)

Lindström J, Peltonen M, Eriksson JG, Louheranta A, Fogelholm M, Uusitupa M \& Tuomilehto J 2006 High-fibre, low-fat diet predicts long-term weight loss and decreased type 2 diabetes risk: the Finnish Diabetes Prevention Study. Diabetologia 49 912-920. (https://doi. org/10.1007/s00125-006-0198-3)

Liu S, Willett WC, Manson JE, Hu FB, Rosner B \& Colditz G 2003 Relation between changes in intakes of dietary fiber and grain products and changes in weight and development of obesity among middle-aged women. American Journal of Clinical Nutrition 78 920-927. (https:// doi.org/10.1093/ajcn/78.5.920)

Lu Y, Fan C, Li P, Lu Y, Chang X \& Qi K 2016 Short chain fatty acids prevent high-fat-diet-induced obesity in mice by regulating $\mathrm{G}$ protein-coupled receptors and gut microbiota. Scientific Reports $\mathbf{6}$ 37589. (https://doi.org/10.1038/srep37589)

Maruta H, Yoshimura Y, Araki A, Kimoto M, Takahashi Y \& Yamashita H 2016 Activation of AMP-activated protein kinase and stimulation of energy metabolism by acetic acid in L6 myotube cells. PLOS ONE 11 e0158055. (https://doi.org/10.1371/journal.pone.0158055)

Murakami Y, Ojima-Kato T, Saburi W, Mori H, Matsui H, Tanabe S \& Suzuki T 2015 Supplemental epilactose prevents metabolic disorders through uncoupling protein-1 induction in the skeletal muscle of mice fed high-fat diets. British Journal of Nutrition 114 1774-1783. (https://doi.org/10.1017/S0007114515003505)

Nedergaard J, Bengtsson T \& Cannon B 2007 Unexpected evidence for active brown adipose tissue in adult humans. American Journal of Physiology: Endocrinology and Metabolism 293 E444-E452. (https://doi. org/10.1152/ajpendo.00691.2006)

Neis EP, van Eijk HM, Lenaerts K, Damink SWO, Blaak EE, Dejong CH \& Rensen SS 2019 Distal versus proximal intestinal short-chain fatty acid release in man. Gut 68 764-765. (https://doi.org/10.1136/ gutjnl-2018-316161)

Prospective Studies Collaboration, Whitlock G, Lewington S, Sherliker P, Clarke R, Emberson J, Halsey J, Qizilbash N, Collins R \& Peto R 2009 Body-mass index and cause-specific mortality in 900000 adults: collaborative analyses of 57 prospective studies. Lancet $\mathbf{3 7 3}$ 1083-1096. (https://doi.org/10.1016/S0140-6736(09)60318-4)

Reynolds A, Mann J, Cummings J, Winter N, Mete E \& Te Morenga L 2019 Carbohydrate quality and human health: a series of systematic reviews and meta-analyses. Lancet 393 434-445. (https://doi. org/10.1016/S0140-6736(18)31809-9)

Sahuri-Arisoylu M, Brody LP, Parkinson JR, Parkes H, Navaratnam N, Miller AD, Thomas EL, Frost G \& Bell JD 2016 Reprogramming of hepatic fat accumulation and 'browning' of adipose tissue by the short-chain fatty acid acetate. International Journal of Obesity $\mathbf{4 0}$ 955-963. (https://doi.org/10.1038/ijo.2016.23)

Scheppach W, Pomare EW, Elia M \& Cummings JH 1991 The contribution of the large intestine to blood acetate in man. Clinical Science 80 177-182. (https://doi.org/10.1042/cs0800177)

Schönfeld P \& Wojtczak L 2016 Short-and medium-chain fatty acids in energy metabolism: the cellular perspective. Journal of Lipid Research 57 943-954. (https://doi.org/10.1194/jlr.R067629)

Stephen AM, Champ MM-J, Cloran SJ, Fleith M, Van Lieshout L, Mejborn H \& Burley VJ 2017 Dietary fibre in Europe: current state of knowledge on definitions, sources, recommendations, intakes and relationships to health. Nutrition Research Reviews 30 149-190. (https://doi.org/10.1017/S095442241700004X)

Tilg H, Moschen AR \& Kaser A 2009 Obesity and the microbiota. Gastroenterology 136 1476-1483. (https://doi.org/10.1053/j. gastro.2009.03.030)

van der Beek CM, Canfora EE, Lenaerts K, Troost FJ, Damink SWMO, Holst JJ, Masclee AAM, Dejong CHC \& Blaak EE 2016 Distal, not proximal, colonic acetate infusions promote fat oxidation and improve metabolic markers in overweight/obese men. Clinical Science 130 2073-2082. (https://doi.org/10.1042/CS20160263)

Williamson DF, Kahn HS, Remington PL \& Anda RF 1990 The 10-year incidence of overweight and major weight gain in US adults. Archives of Internal Medicine 150 665-672.

Yamashita H, Maruta H, Jozuka M, Kimura R, Iwabuchi H, Yamato M, Saito T, Fujisawa K, Takahashi Y, Kimoto M, et al. 2009 Effects of acetate on lipid metabolism in muscles and adipose tissues of type 2 diabetic Otsuka Long-Evans Tokushima Fatty (OLETF) rats. Bioscience, Biotechnology, and Biochemistry 73 570-576. (https://doi.org/10.1271/ bbb.80634)

Received in final form 24 April 2019

Accepted 1 May 2019

Accepted Preprint published online 1 May 2019 https://joe.bioscientifica.com https://doi.org/10.1530/JOE-19-0098 (c) 2019 Society for Endocrinology Published by Bioscientifica Ltd. Printed in Great Britain 